\title{
Coronary stent symmetry and vascular injury determine experimental restenosis
}

\author{
C Schulz, R A Herrmann, C Beilharz, J Pasquantonio, E Alt
}

\begin{abstract}
Objective-To assess the impact of stent symmetry on restenosis using the coronary overstretch sheep model.

Methods-Neointimal thickness, injury index, and percentage diameter and area stenosis were calculated by digital morphometry. The standard deviation of the angular burden was used to assess stent symmetry for each section.

Materials-15 healthy Merino sheep (63-75 kg) underwent implantation of 30 slotted tube stents $(7 \mathrm{~mm})$. Restenosis was induced by calculated overstretch of the coronary artery. Twenty eight days after implantation, stents were excised and underwent histological examination using quantitative digital morphometry.

Results-The severity of vessel injury was positively correlated with neointimal thickness and with percentage diameter and area stenosis $(\mathrm{p}<0.001)$. Mean neointimal thickness and mean vascular injury per cross section were strongly related to the standard deviation of angular burden, with correlation coefficients of 0.6 and 0.8 , respectively $(\mathrm{p}<0.001)$.

Conclusions-The well known relation between vascular injury and restenosis was confirmed, and a new relation was discovered between stent asymmetry and restenosis. If these results apply to human coronary arteries, restenosis may also be dependent on the degree of asymmetric stent expansion. These results should influence the development of new stent designs to reduce asymmetric stent expansion, leading to a more homogeneous strain distribution in stented coronary segments.
\end{abstract}

(Heart 2000;83:462-467)

Keywords: stent symmetry; vascular injury; restenosis; interventional cardiology

Compared with balloon angioplasty alone, a lower restenosis rate has been found with the use of slotted tube stents. ${ }^{12}$ The beneficial effects of stent implantation are attributed to a large acute lumen gain when compared with balloon angioplasty alone, and a significant reduction in vessel recoil after intervention. ${ }^{3-5}$ More recently the beneficial effects of coronary stent implantation on the prevention of late remodelling have been demonstrated. ${ }^{67}$ When angioplasty alone is carried out, remodelling seems to play a critical role in the development of restenosis. ${ }^{8}$ However, stents have failed to improve the neointimal proliferative response to vessel injury that is seen with both balloon angioplasty and in stented vascular segments. $^{910}$ This proliferative response after coronary stenting has been attributed to stent induced vascular injury, because there is a proportional relation between the degree of injury and neointimal proliferation. ${ }^{11}$ Furthermore, the permanent strain on the vessel wall after stent implantation, compared with the transient strain after balloon angioplasty, may result in a prolonged stimulus for neointimal formation. Grinstead et $a{ }^{12}{ }^{12}$ using a balloon expandable coil stent, reported significant adventitial injury 28 days after stent deployment in pig coronary arteries, with a mean stent to vessel ratio of $1.06: 1$. Similar findings were reported in another study by Holmes et $a l,{ }^{13}$ using a tantalum coil stent. The authors attributed the severe arterial injury to chronic stent-vessel wall interactions, as the balloon inflated diameters were selected to match the vessel diameter. In a recent study Hofma et al showed that vessel injury increased progressively up to 12 weeks after stent implantation, and this was not seen after balloon angioplasty alone. ${ }^{14}$ Furthermore differences in the degree of acute vessel injury were dependent on stent design, which was confirmed on longer follow up. ${ }^{14}$

Future trends in stent design will involve fewer strut to strut intersections, multicellular designs, and increased flexibility, with the aim of reducing stent-vessel wall interactions and restenosis. ${ }^{15}{ }^{16}$ However, if vascular strain is a factor in restenosis, this must be taken into consideration in stent design, as local strain is strongly determined by the amount of vessel wall supported by each stent strut. This becomes crucial if one observes the asymmetrical stent expansion possible with coronary stents, both in vitro and in vivo. We therefore designed a study to investigate a possible association between asymmetric stent expansion, vascular injury, and restenosis, using a coronary overstretch sheep model. The purpose of the study was to investigate whether such a relation exists, and if so, to quantify the influence of vessel injury and stent symmetry on restenosis.

\section{Methods}

SURGICAL PROCEDURE

The study was performed on adult Merino sheep, purchased from a single local farm. Animal research was approved by the institutional review board and the animal use committee. 
The guidelines for animal investigation are equivalent to the Position of the American Heart Association on research and animal use. Fifteen healthy sheep (63-75 kg) on a normal diet underwent placement of 30 commercially available $7 \mathrm{~mm}$ slotted tube stents (PalmazSchatz stents, Johnson and Johnson, International Systems, Warren, New Jersey, USA) in the left anterior descending or left circumflex coronary arteries. Under general anaesthesia, a $7 \mathrm{~F}$ sheath was placed retrogradely in the left carotid artery, while jugular vein access was obtained for drug delivery. Animals were treated intravenously with $500 \mathrm{mg}$ aspirin and $300 \mathrm{mg}$ amiodarone. Heparin (100 U/kg) was given intravenously immediately after sheath placement to achieve an activated partial thromboplastin time (aPTT) of more than 180 seconds. The stents were implanted using the guiding catheter as a reference, in order to obtain a 1.3:1 balloon to vessel ratio compared with the baseline vessel diameter. The stents were hand crimped onto PTCA balloons as used for coronary angioplasty (Viva, Boston Scientific Corporation, Galway, Republic of Ireland), with balloon diameters from 2.5 to $4.0 \mathrm{~mm}$. Placement was achieved with a single balloon inflation at 8-12 atm for 30 seconds. Angiography was completed after implantation to confirm patency of the stent and side branches, as well as to assess stent migration and intraluminal filling defects. At the end of the procedure, the carotid sheath was removed and the artery sutured. The neck wound was closed with interrupted sutures. Animals were allowed to recover and returned to care facilities. The animals returned for follow up coronary angiography at day 28 after implantation, after which they were killed with an overdose of pentobarbitone (pentobarbital).

QUANTITATIVE ANGIOGRAPHY

Quantitative angiography analysis was performed with an automated edge detection system (Medis, Medical Imaging Systems, Nuenen, Netherlands). The guiding catheter tip was used for calibration. Mean baseline diameter and the mean diameter of the maximally inflated balloon during stent placement were obtained from this analysis system. Balloon to vessel ratio was calculated as mean diameter of the inflated balloon divided by the coronary baseline diameter. At follow up, minimum lumen diameter and reference diameter were used to calculate percentage diameter stenosis.

\section{HISTOLOGICAL EVALUATION}

Immediately following euthanasia, the heart and ascending aorta were excised. The coronary arteries were perfusion fixed with $10 \%$ neutral buffered formalin at $100 \mathrm{~mm} \mathrm{Hg}$ for 15 minutes from the aortic stump. The stented coronary arteries were carefully dissected from the epicardial surface and left in $10 \%$ formalin overnight. After dehydration and infiltration sections were embedded in methylmethacrylate. Sections of $100 \mu \mathrm{m}$ thickness were obtained from four different levels of the stented segment, using a slow rotating diamond saw. These sections were mounted on slides and polished to an approximate thickness of 50 to $70 \mu \mathrm{m}$. All histological sections were stained with Paragon stain. The cross sectional area of each section was measured with digital morphometry (NIH Image 1.59 for quantitative analysis) to determine the areas within the internal elastic lamina (IEL) and the vessel lumen. The percentage area stenosis was then defined as: (IEL - lumen area/IEL $\times 100$ ). Total neointimal area was calculated as the difference between original and stenotic lumen (original area minus stenotic area). Neointimal thickness extending perpendicularly to the lumen was measured at each strut site. The severity of stent induced vascular injury was graded according to the method of Schwartz et al. ${ }^{11}$ In brief, the degree of arterial injury at each stent strut site was assessed as:

Grade 0-Internal elastic lamina intact with media compressed;

Grade 1-Internal elastic lamina lacerated with media compressed;

Grade 2-Internal elastic lamina and media lacerated with external elastic lamina intact; Grade 3-External elastic lamina lacerated.

The mean injury score for each section was calculated by dividing the sum of injury scores at each strut site by the total number of struts from the section. Angular burden was used as a measurement of the unsupported vessel wall between struts relative to the overall circumference expressed in degrees:

$$
\mathrm{AB}=\left(0.5 \mathrm{~d}_{1}+0.5 \mathrm{~d}_{2} / \mathrm{C}\right) \times 360^{\circ}
$$

where $A B$ is angular burden, $d_{1}$ and $d_{2}$ are the distances from the strut to each neighbouring strut, and C is total stent circumference. Stent symmetry per cross section was assessed by calculating the standard deviation of angular burden.

SCANNING ELECTRON MICROSCOPY

The stents from two sheep were used for scanning electron microscopy. The excised stents were rinsed in three changes of sodium phosphate for 15 minutes each, then fixed in $1 \%$ glutaraldehyde overnight and rinsed in distilled water. Specimens were then dehydrated in a series of graded ethanols (50-100\%). After the critical point of drying, the specimens were mounted, placed in a vacuum coater, and coated with a 30-40 nm layer of gold for scanning electron microscopy (Zeiss, AMR 1200).

\section{STATISTICAL ANALYSIS}

All data are presented as mean (SD). For each of the sections investigated, mean injury score, mean neointimal thickness, total neointimal area, percentage diameter and area stenosis, and standard deviation of angular burden were calculated. Gaussian distribution of the standard deviation of angular burden was tested using the Kolmogorov-Smirnov (K-S) test. Simple linear regression was performed by using standard methods to derive a slope, intercept, and correlation coefficient. Significance of the relations was tested by analysis of variance. A $p$ value $<0.05$ was considered significant. Computations were performed with the Sigma Stat 2.0 statistical system. 


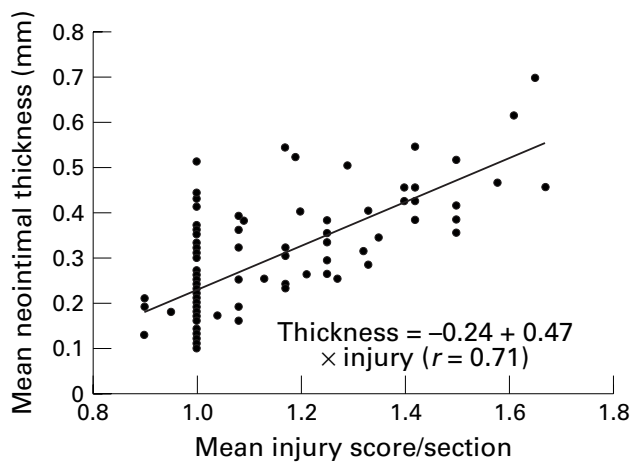

Figure 1 Scatterplot of mean vascular injury score versus mean neointimal thickness per section. There is a proportional relation. The scatter increases for increasing depth of injury.

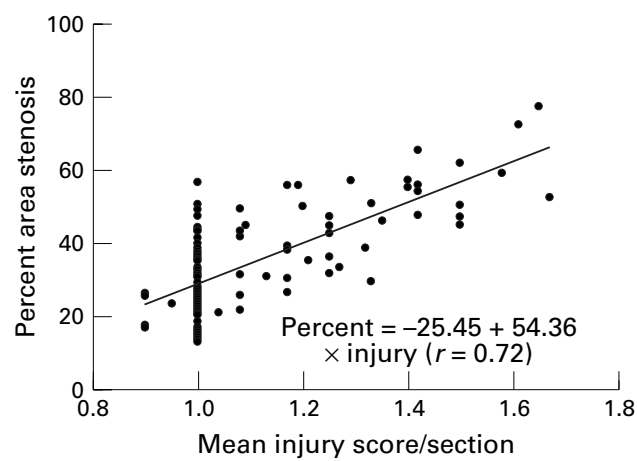

Figure 2 Scatterplot of mean vascular injury versus percentage area stenosis per section. There is a proportional relation, as in fig 1 .

\section{Results}

STENT IMPLANTATION

Thirty slotted tube stents were successfully implanted in the left anterior $(n=15)$ and left circumflex $(n=15)$ coronary arteries of 15 Merino sheep. All sheep survived without problems until death was induced. Quantitative analysis of the coronary angiograms at implant revealed a mean (SD) balloon to vessel ratio of $1.3(0.2)$. Average balloon diameter and balloon pressure were $3.2(0.2) \mathrm{mm}$ and 8.5 (1.5) atm, respectively.

HISTOLOGICAL EXAMINATION

Coronary histology at 28 days after stent placement showed a neointima consisting of a dense circumferentially arranged population of smooth muscle cells on the lumen surface, with less well organised spindle shaped cells and rare multinuclear giant cells in the region of the struts. Microscopic examination revealed neointimal responses of different magnitudes. The mean neointimal thickness at each strut site was on average $380(180) \mu \mathrm{m}$. The area of the lumen within the stent was $4.24(1.33) \mathrm{mm}^{2}$; the percentage diameter stenosis was 19.69 (9.00)\% (range $6.81 \%$ to $51.94 \%$ ), and the percentage area stenosis, $34.62(13.81) \%$

Table 1 Regression and correlation with vessel injury score

\begin{tabular}{lccll}
\hline & Slope & Intercept & r Value & $p$ Value \\
\hline Neointimal thickness $(\mathrm{mm})$ & 0.47 & -0.25 & 0.71 & $<0.001$ \\
Per cent diameter stenosis & 36.00 & -20.1 & 0.73 & $<0.001$ \\
Per cent area stenosis & 54.36 & -25.45 & 0.72 & $<0.001$ \\
\hline
\end{tabular}

(range $13.16 \%$ to $76.91 \%$ ). Figures 1 and 2 show plots of mean vessel injury score versus neointimal thickness and percentage area stenosis for each injured segment. Linear regression analysis showed that there was a significant positive correlation between the mean injury index and the percentage diameter and percentage area stenosis. Table 1 shows the regression variables of slope, intercept, Pearson correlation coefficient, and $\mathrm{p}$ value for each of these relations.

The normality test was passed for the standard deviation of angular burden (K-S distance $=0.081, \mathrm{p}=0.112$ ). Stent symmetry was assessed on a per section basis, which showed a positive correlation among the average injury score, neointimal thickness, and the standard deviation of the angular burden (fig 3, A and B, fig 4, and fig 5). Regression variables of slope, intercept, correlation coefficient, and $\mathrm{p}$ values are listed in table 2.

Scanning electron microscopy showed spindle shaped endothelial cells oriented in the direction of flow, covering more than $95 \%$ of the luminal surface. However, badly torqued struts could be seen close to strut intersections in areas with pronounced stent extension (fig 6).

\section{Discussion}

Restenosis after coronary stent placement remains a problem of major concern. Although stent implantation results in larger immediate luminal diameters than balloon angioplasty, stents also provoke an exaggerated proliferative response within the vascular wall, resulting in excessive neointima formation compared with balloon induced vessel injury. This process is reflected by the significantly greater late loss of minimum lumen diameter seen in STRESS (Stent restenosis study) and BENESTENT (Belgium-Netherlands stent study), which substantially mitigated the initial angiographic advantages of stenting. ${ }^{17} 18$

VESSEL INJURY AND NEOINTIMAL PROLIFERATION Schwartz et al first reported the nature of the relation between stent induced arterial injury and neointimal proliferation using the porcine coronary overstretch model. ${ }^{11}$ They were able to show a proportional relation between the vessel injury and neointimal proliferation. They suggested that this may also hold true for human restenosis. Correspondingly, for the balloon expandable articulated Palmaz-Schatz stent, with a bridging strut between two shorter stainless steel slotted tube stents, it could be shown that in restenotic lesions involvement of the articulation or stent edges occurred in $83 \%$ of all lesions, together with a greater loss index. ${ }^{19}$ The investigators believed that this was mainly because the edges of the slotted tube stent tended to dilate more than the body during normal inflation. Thus the edges of the stent were embedded more deeply in the vessel wall than the body. Our results are in agreement with those findings (figs 1 and 2). In our study a mean neointimal thickness of 380 (180) $\mu \mathrm{m}$ was found, with a strong correlation between neointimal thickness and increased mean injury score. It can be concluded that 

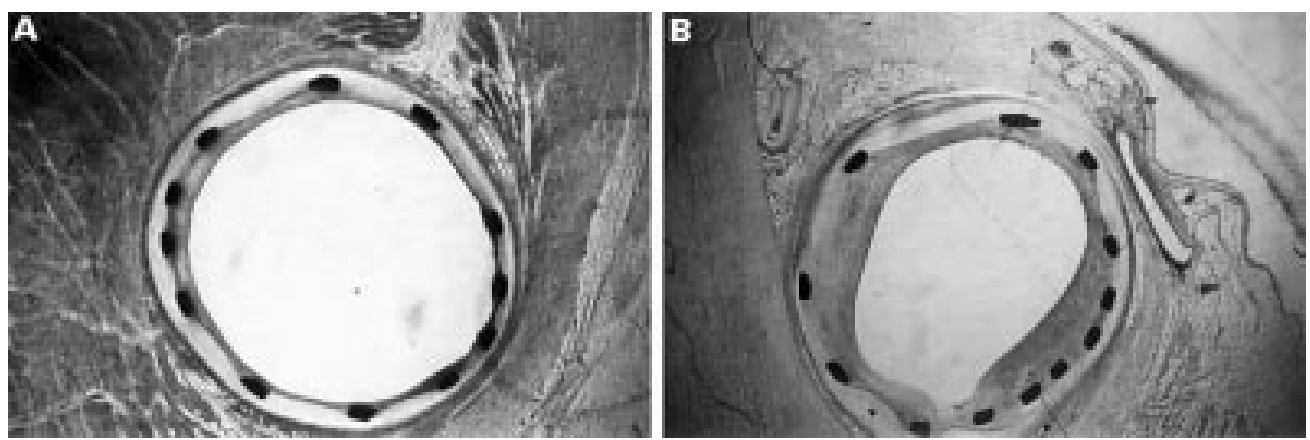

Figure 3 Low power photomicrographs from methylmethacrylate embedded sections from sheep coronary arteries 28 days after stent implantation. (Paragon stain, magnification $\times 14$.) Representative cross section with symmetric $(A)$ and asymmetric (B) stent expansion. An eccentric and more pronounced neointima formation is present in $(B)$ which can be attributed to asymmetric stent expansion.

deep arterial laceration causes the thickest neointima and more severely compromises the lumen. Milder vessel trauma may result in less neointimal thickness and less lumen stenosis. It is tempting to extrapolate these findings to the clinical situation, with the concerns about a potential exacerbation of the hyperplastic response from the vessel wall with the use of high balloon pressures. This, however, may not be the case, as high pressure balloon inflation with pressures up to 24 atm was shown to result in a reduction in the incidence of thrombotic events without an increase in the restenosis rate. ${ }^{20}{ }^{21}$ Furthermore, in a prospective randomised study using 934 consecutive patients assigned to either high pressure (15-20 atm) or low pressure (8-13 atm) coronary stent

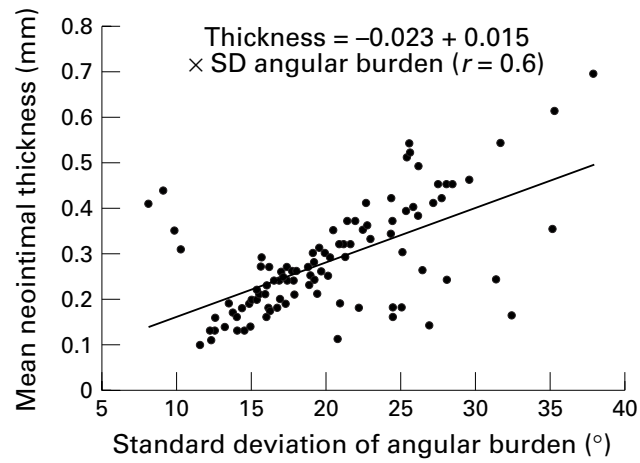

Figure 4 Scatterplot of standard deviation of angular burden versus mean neointimal thickness per section. The proportional relation indicates that restenosis is related to asymmetric stent expansion.

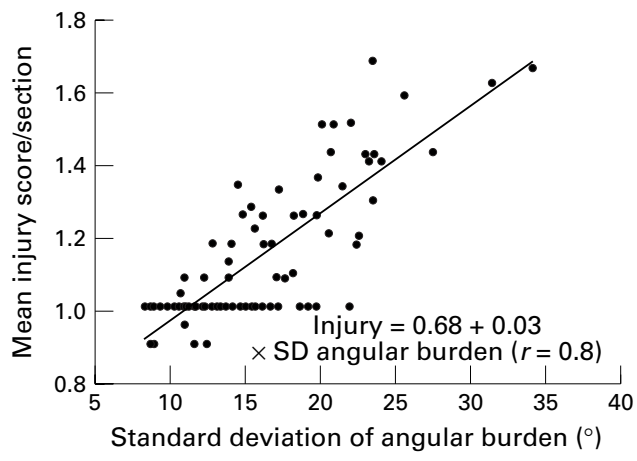

Figure 5 Scatterplot of standard deviation of angular burden versus average injury score per section. The injury vessel score is related to the standard deviation of the angular burden, showing that asymmetric stent deployment leads to increased vascular injury. implantation, it could be shown that restenosis is not a function of the balloon pressure strategy employed. ${ }^{22}$ Most importantly, the rate of adverse clinical events over one year after the intervention was comparable for both treatment strategies. In fact, the final lumen achieved after stent implantation is one of the most powerful predictors of restenosis. ${ }^{23}$ Thus high pressure dilatation may also have a positive influence on restenosis as a result of the better lumen achieved.

IMPACT OF STENT ASYMMETRY ON NEOINTIMAL THICKNESS AND VASCULAR INJURY

Confirmation of successful stent implantation is based on the angiographic appearances. A stent implantation procedure can be regarded as successful if the following criteria are met: a smooth contour of the lumen silhouette in the stented segment; diameter stenosis inside the stent in the worst (of at least two orthogonal) views $<15 \%$ by quantitative on line analysis; and no inflow or outflow obstruction. ${ }^{24}$ However, angiographic projection imaging may not reveal the three dimensional geometry that is necessary to appreciate the full and homogeneous expansion of a cylindrical meshwork device such as an intracoronary stent. Furthermore, close contact between the stent struts and the vessel wall and symmetrical stent expansion cannot be confirmed by angiography. Despite adequate angiographic results, Nakamura et al showed a high frequency of incompletely and asymmetrically expanded stents using intravascular ultrasound imaging. ${ }^{25}$ It appeared that the less diseased perimeter of the vessel wall expanded more than the portion of the vessel involved with dense fibrocalcific plaque. ${ }^{25}$ However, in a recent study where serial intravascular ultrasound was performed after intervention and at follow up on 142 slotted tube stents in 115 lesions, even when neointimal tissue accumulation was normalised for postintervention lumen cross sectional area stent symmetry did not correlate with follow up neointimal tissue accumulation, either within the stent segment or downstream of it. ${ }^{6}$ Furthermore, the worst asymmetry index within an individual stent did not correlate with overall neointimal tissue accumulation. These data might suggest that symmetry has no impact on neointimal proliferation within stented coronary segments. However, with 
Table 2 Regression and correlation with standard deviation of angular burden

\begin{tabular}{|c|c|c|c|c|}
\hline & Slope & Intercept & $r$ Value & p Value \\
\hline Neointimal thickness (mm) & 0.012 & 0.045 & 0.60 & $<0.001$ \\
\hline Injury score & 0.029 & 0.68 & 0.80 & $<0.001$ \\
\hline
\end{tabular}

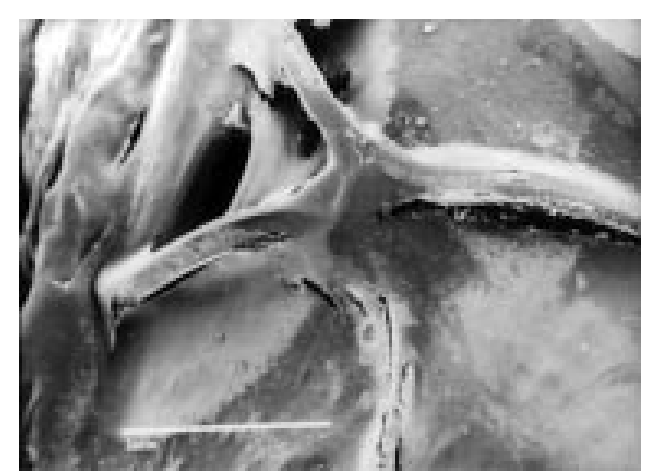

Figure 6 Scanning electron microscopy $(\times 27.5$

magnification) showing a strut intersection of a slotted tube stent 28 days after implantation in a sheep coronary artery. Note the badly torqued struts in that area of pronounced stent extension.

intravascular ultrasound it should be remembered that stent symmetry index is calculated from the minimum and maximum stent diameters (stent symmetry $=$ minimum stent diameter/maximum stent diameter), and this does not necessarily correlate with the local stent induced strain.

In the present study the standard deviation of the angular burden was used as a measure of the stent symmetry and local vessel wall strain. Measurements were performed on a strut by strut basis, giving information about the open vessel wall supported by each strut. The standard deviation of the angular burden showed a significant correlation with vascular injury and local neointimal thickness. This correlation was as strong as that between neointimal thickness and injury. As angular burden seems also to be a measure of local vessel wall strain, it is possible that this local strain is as important a factor in neointimal formation as injury. Thus our study has illuminated further the quantitative evidence that vessel symmetry is an additional or related factor in neointimal hyperplasia. Scanning electron microscopy showed highly torqued struts close to strut intersections, especially if a pronounced local stent extension was seen, exposing the sharp strut edges to the vessel wall (fig 6). These findings might represent the morphological correlate between stent asymmetry (for example, the standard deviation of the angular burden) and neointimal thickness.

In addition, local stretching of the vessel wall has been identified as an important factor in pronounced neointimal proliferation. This can be attributed to increased mechanical deformation and stretching of putative smooth muscle cells in the arterial wall, leading to activation of signalling pathways and cell replication, as has been described in different in vitro and in vivo models. ${ }^{18} 2627$ Cheng et al reported that even a transient mechanical stimulus increased DNA synthesis in human vascular smooth muscle cells. ${ }^{27}$ The cell response to the mechanical stimulus was mainly due to autocrine and paracrine release of fibroblast growth factor-2 (FGF-2). Later work from the same group confirmed that cellular strain closely modulates the release of intracellular FGF-2 from human vascular smooth muscle cells. ${ }^{28}$ Furthermore, FGF-2 release was negligible in response to smaller amounts of strain. Hence, a biphasic vascular response would be understandable, which was not seen in the present study. One might argue that the stent/balloon induced vascular stress profile exceeded the threshold value throughout the entire vessel wall, precluding a biphasic vascular response. In accord with these observations it was shown that balloon catheter injury of the rat carotid is linked to activation of the extracellular signal regulated kinase $1 / 2$ (ERK 1/2) pathway, which could be inhibited by adding an FGF-2 antibody. ${ }^{18}$ Several lines of evidence suggests that mechanotransduction through the extracellular matrix may regulate the expression of matrix metalloproteinases (MMP) ${ }^{29}$ MMP may play a critical role in tissue repair and remodelling. However, the expression of endogenous tissue inhibitors of MMP (TIMP) also increases dramatically during neointima formation. ${ }^{30}$ Thus neointima formation may in part represent the balance between MMP and TIMP expression. Furthermore, a significant increase within the first few weeks after injury has been shown for platelet derived growth factor (PDGF), transforming growth factor $\beta$, and angiotensin II. ${ }^{26}$ However, the extent to which increased and prolonged local vascular strain induces the release of these growth factors is still a matter of debate. It could be speculated that similar mechanisms are activated after stent implantation, which are at least partially regulated by local vascular strain; this could explain the results obtained in the present study.

\section{STUDY LIMITATIONS}

Our study was performed on healthy animals, so the effects in atherosclerotic vessels could not be directly assessed. As this study used slotted tube Palmaz-Schatz stents, it is uncertain whether the results can be applied to other stent designs. However, as vascular strain would occur in all stent designs, it can be assumed that the results are relevant to other stent designs as well.

\section{CONCLUSIONS}

The well known relation between vessel injury and restenosis was confirmed, and a new relation discovered between vessel strain/symmetry and restenosis. New stent designs which focus on addressing both the mechanical and biochemical causes of late lumen loss are likely to improve long term outcome of patients after coronary artery stenting. Future developments in coronary scaffolding devices should focus on improved stent symmetry in order to apply a more homogeneous strain distribution in stented coronary segments and to prevent the neointimal hyperplasia that is presumably the main mechanism of late lumen renarrowing. 
1 Fischman DL, Leon MB, Baim DS, et al. A randomized comparison of coronary-stent placement and balloon Engl f Med 1994;331:496-501.

2 Serruys PW, de Jaegere P, Kiemeneij F, et al. A comparison of balloon-expandable-stent implantation with balloon angioplasty in patients with coronary artery disease. $N$ Engl 7 Med 1994;331:489-95.

3 Kuntz RE, Gibson CM, Nobuyoshi M, et al. Generalized model of restenosis after conventional balloon angioplasty, stenting and directional atherectomy. $\mathcal{F} \mathrm{Am}$ Coll Cardio 1993;21:15-25.

4 Kuntz RE, Safian RD, Carrozza JP, et al. The importance of acute luminal diameter in determining restenosis after coronary atherectomy or stenting. Circulation 1992;86:182735.

5 Haude M, Erbel R, Issa H, et al. Quantitative analysis of elastic recoil after balloon angioplasty and after intracoronary implantation of balloon-expandab

6 Hoffmann R, Mintz GS, Dussaillant GR, et al. Patterns and Hoffmann R, Mintz GS, Dussaillant GR, et al. Patterns and mechanisms of in-stent restenosis: a serial in

7 Painter JA, Mintz GS, Wong SC, et al. Serial intravascular ultrasound studies fail to show evidence of chronic PalmazSchatz stent recoil. Am f Cardiol 1995;75:398-400.

8 Andersen HR, Maeng M, Thorwest M, et al. Remodeling rather than neointimal formation explains luminal narrowin after deep vessel wall injury. Circulation 1996;93:1716-24.

9 Dussaillant GR, Mintz GS, Pichard AD, et al. Small stent size and intimal hyperplasia contribute to restenosis: a volumetric intravascular ultrasound analysis. $\mathcal{f} \mathrm{Am} \mathrm{Coll}$ Cardiol 1995;26:720-4.

10 Gordon PC, Gibson CM, Cohen DJ, et al. Mechanisms of restenosis and redilation within coronary stentsquantitative angiographic assessment. $\mathcal{f} \mathrm{Am}$ Coll Cardiol 1993;21:1166-74.

11 Schwartz RS, Huber KC, Murphy JG, et al. Restenosis and the proportional neointimal response to coronary artery injury: results

12 Grinstead WC, Rodgers GP, Mazur W, et al. Comparison of three porcine restenosis models: the relative importance of hypercholesterolemia, endothelial abrasion, and stenting Coron Artery Dis 1994;5:425-34.

13 Holmes DR, Camrud AR, Jorgenson MA, et al. Polymeric stenting in the porcine coronary artery model: differentia outcome of exogenous fibrin sleeves versus polyurethanecoated stents. F Am Coll Cardiol 1994;24:525-31.

14 Hofma SH, Whelan DMC, van Beusekom HMM, et al. Increasing arterial wall injury after long-term implantation of two types of stent in a porcine coronary model. Eur Heart f 1998;19:601-9.

15 Thomas WJ, Laird JR, Bailey L, et al. Experimental results with the MULTI-LINK ${ }^{\mathrm{TM}}$ stent in a porcine model. F Invas Cardiol 1997;9:453-60.
16 Rogers C, Edelman ER. Endovascular stent design dictates experimental restenosis and thrombosis. Circulation 1995; 91:2995-3001.

17 Narins CR, Holmes DR, Topol EJ. A call for provisional stenting. Circulation 1998;97:1298-305.

18 Koyama H, Olson NE, Dastvan FF, et al. Cell replication in the arterial wall. Circ Res 1998;82:713-21.

19 Ikari Y, Hara K, Tamura T, et al. Luminal loss and site of restenosis after Palmaz-Schatz coronary stent implantation. Am $\mathcal{F}$ Cardiol 1995;76:117-20.

20 Waksman R, Shen Y, Ghazzi Z, et al. Optimal balloon inflation pressures for stent deployment and correlates of stent thrombosis and in-stent restenosis [abstract]. Circulation 1996;94(suppl):I-258.

21 Goldberg SL, Di Mario C, Hall P, et al. Comparison of aggressive versus nonagressive balloon dilatation for stent deployment on late loss and restenosis in native coronary arteries. Am f Cardiol 1998;81:708-12.

22 Dirschinger J, Kastrati A, Neumann FJ, et al. Influence of balloon pressure during stent placement in native coronary arteries on early and late angiographic and clinical outcome: a randomized evaluation of high pressure inflation. Circulation 1999;100:918-23.

23 Kastrati A, Schömig A, Elezi S, et al. Predictive factors of restenosis after coronary stent placement. $\mathcal{F} \mathrm{Am}$ Coll Cardiol 1997;30:1428-36

24 von Birgelen C, Mintz GS, Nicosia A, et al. Electrocardiogram-gated intravascular ultrasound image acquisition after coronary stent deployment facilitates on-line three-dimensional reconstruction and automated lumen quantification. $\mathcal{F}$ Am Coll Cardiol 1997;30:436-43.

25 Nakamura S, Colombo A, Gaglione A, et al. Intracoronary ultrasound observations during stent implantation. Circulation 1994;89:2026-34

26 Nikol S, Huehns TY, Hofling B. Molecular biology and post-angioplasty restenosis. Atherosclerosis 1996;123:17-31.

27 Cheng GC, Libby P, Grodzinsky AJ, et al. Induction of DNA synthesis by a single transient mechanical stimulus of human vascular smooth muscle cells. Circulation 1996;93: human

28 Cheng GC, Briggs WH, Gerson DS, et al. Mechanical strain tightly controls fibroblast growth factor- 2 release from cultured human vascular smooth muscle cells. Circ Res 1997; 80:28-36.

29 Yang JH, Briggs WH, Libby $\mathrm{P}$, et al. Small mechanical strains selectively suppress matrix metalloproteinase-1 expression by human vascular smooth muscle cells. $\mathcal{F}$ Biol Chem 1998;273:6550-5.

30 Kranzhöfer A, Baker AH, George SJ, et al. Expression of tissue inhibitor of metalloproteinase-1, -2 , and -3 during neointima formation in organ cultures of human saphenous vein. Arterioscler Thromb Vasc Biol 1999;19:255-65. 